\title{
Lightweight VNF Manager Solution for Virtual Functions
}

\author{
Wang $\mathrm{Bo}^{1}$ and Odini Marie-Paule ${ }^{2}$ \\ ${ }^{1}$ ES CMS. Hewlett Packard, Shanghai, China \\ ${ }^{2}$ ES CMS. Hewlett Packard, Grenoble, France \\ E-mail: \{bow; marie-paule.odini\}@hp.com
}

Received 09 October 2014;

Accepted 01 May 2015

\begin{abstract}
This paper presents a creative lightweight solution to package any existing VNF (Virtualized Network Function) to support ETSI NFV (Network Function Virtualization) architecture quickly and easily. The preconditions of existing VNF are quite straightforward and simple, such as running on Virtual Machine (VM) and Operating System (OS) supporting Secure Shell (SSH) commands. With our solution, the new VNF (Virtualized Network Function) will have the additional functions required to integrate with ETSI NFV architecture, without redesigning the existing Element Manager. This will include VNF Descriptor, lifecycle management benefits such as deploy within minutes, scale out/in on demand, etc.
\end{abstract}

Keywords: NFV, VNF, VNFM, EM.

\section{Problem Statement}

Networks Functions Virtualization (NFV) is a powerful emerging technique with widespread applicability. It is about virtualizing network functions, leveraging general purpose platforms (Compute, Network, and Storage) and virtualization to provide agility, flexibility and decouple network functions from physical resources. It enables CSPs (Communication Service Providers)

Journal of ICT, Vol. 3, 91-104.

doi: $10.13052 /$ jicts2245-800X.315

(c) 2015 River Publishers. All rights reserved. 
to radically take down infrastructure capital and operational costs - and provide a more agile environment to introduce new services more quickly at far less cost than previous network infrastructure. The main business benefits includes Cost/ROI, Business agility, Scalability, Flexibility and Innovation. ETSI (European Telecommunications Standard Institute) is working on NFV and has released the first phase specifications [1].

To implement NFV, a Network Function (NF), which is today often a network node or physical appliance and its Element Manager (EM, a component to manage the NF), has to be virtualized as a VNF (Virtualized Network Function). NFV decouples software implementations of Network Functions from the compute, storage, and networking resources they are using. It adds new capabilities to communications networks and requires a new set of management and orchestration functions to be added to the current model of operations, administration, maintenance and provisioning. It requires VNF to provide not only an EM (Element Manager) to take care of the configuration and management of the network functions, but also a VNF Manager. The VNF manager typically takes care of the lifecycle of the VNF. It is tightly coupled to the service logic of the VNF and this intelligence typically comes from the VNF vendor.

HP has multiple telecommunication products, which acts as NFV NF (Network Function). Most of these products are evolving to NFV, and support at least HP NFV Level 2, which means run on a VM. In parallel, HP has a product, NFV Director (NFV-D), for NFV Orchestrator, that embeds a default generic VNF Manager. But no solution exist for VNF level 2 to be sold to customers that have an existing NFV Orchestrator and only want HP VNF and that VNF EM+VNF Manager. Two options have been considered:

First, evolving existing EM (Element Manager) or having VNF team develop a VNF Manager for its product. But this solution does not seem to make sense: it would mean a duplication of effort for each VNF team, time consuming and costly; Some NF have rich management features, but evolving this to NFV may be a challenge for the teams: not all of these features are required for NFV, and it may be difficult to modify the EM code; Other NFs have a lack of management features, and just have some basic capability like CLI. In this case a complete new design is required with often little experience in that team to develop management platforms.

Second, use the NFV-D and its embedded VNF Manager for each product line NF. But this solution was not available when we started the POC (Proof of Concept), and in any case would only work if the customer is ready to 
buy an integrated solution from HP, with VNF and NFV orchestrator/VNF manager. In case another vendor NFV Orchestrator has been deployed in the CSPs' NFV infrastructure. HP VNF will have to integrate with that NFV Orchestrator.

Besides, today there is not a standard and comprehensive VNF Descriptor to describe a VNF in terms of its deployment and operational behavior. ETSI has defined some specifications for descriptors but it remains guidelines not detailed specifications ready for implementation and it is primarily focused on VNF on-boarding and managing the lifecycle of a VNF instance.

In the meantime, CSPs are moving full speed on NFV, with expectations of reducing cost and increasing agility quickly and expect a lightweight solution to demonstrate some VNF's capability in their existing NFV infrastructure.

\section{Our Solution}

Our proposed solution is to provide a lightweight application, which is called VNEM (Virtualized Network function Element Manager) that would be open enough to be used by any VNF (Virtualized Network Function). It provides a VNF independent approach to integrate with any existing VNFs without a new EM (Element Manager) development, and also provide a VNF Manager functionality to support de facto standards Openstack with the NFV infrastructure. Figure 1 illustrates the VNEM position in ETSI NFV architecture.

The EM (Element Manager) features, which is required in NFV MANO (Management and Orchestration) architecture, are packaged to VNEM as some artifacts, include some XML based workflow configurations, shell script template, cloud-init template, etc.

For the purpose of this POC (Proof of Concept), we have implemented VNEM for the virtualization of an HP Product called HP OCS (Online Charging System). Figure 2 illustrates the functional architecture of VNEM. It is composed of:

- A STF (SSH and Template based Flow engine), which provides the easy integration with any VNF which has SSH capability; all of the VNF context, such as VNF Descriptor (VNFD), VNF Record (VM address, network ...) information, are available for the template, and rendered before SSH interaction with VNF Components. This approach is widely supported by any OS, which enables our solution to integrate with any existing Network Function easily. 
94 W. Bo and O. Marie-Paule

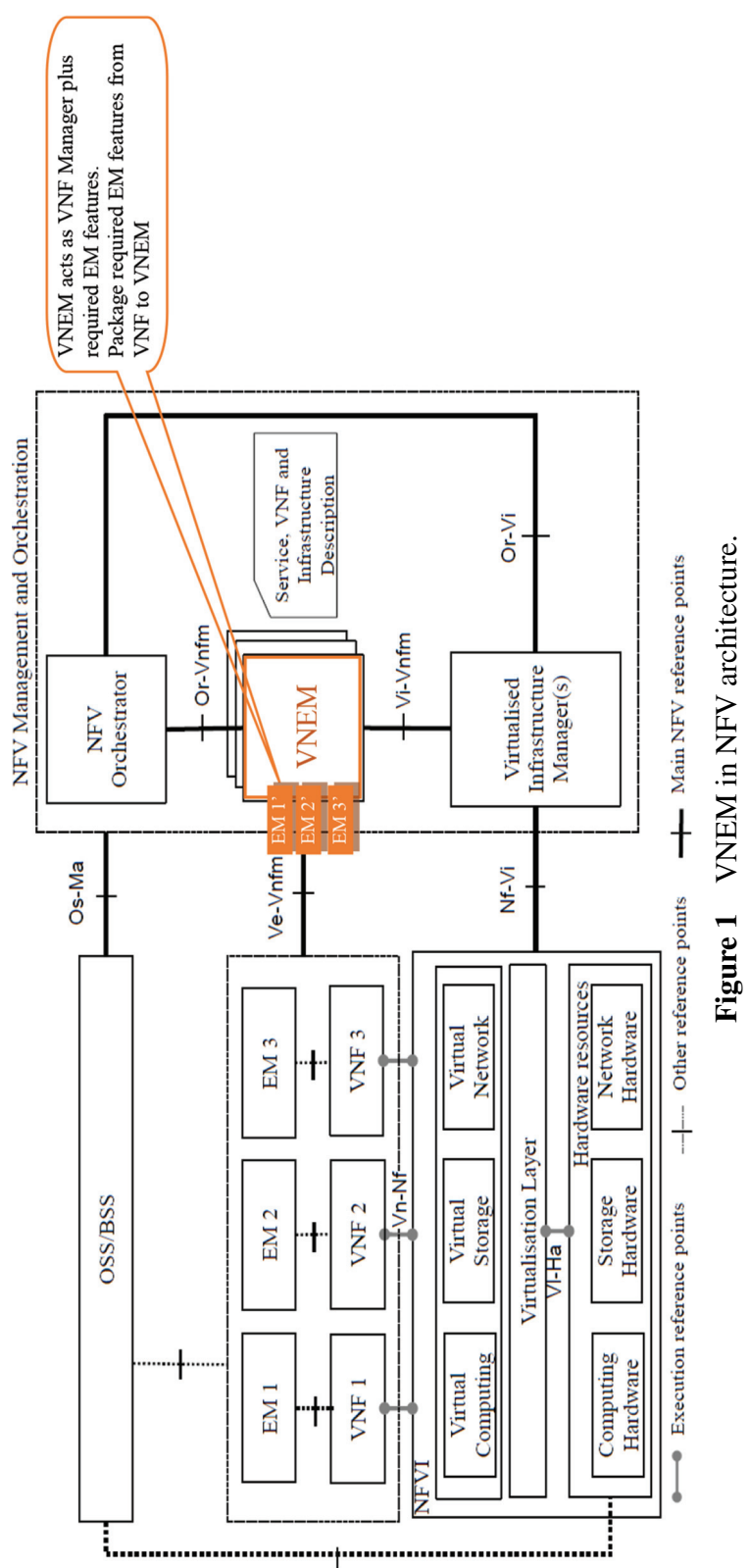







- An Openstack integration plugin with VIM (Virtualized Infrastructure Manager). The VIM in this POC is HP Helion [2], and the Openstack API is de facto standards for VIM integration.

- A resource workflow plugin to compose the VIM interaction tasks. The task types include remote shell, remote copy, and openstack operations.

- A comprehensive and ETSI NFV compliant VNFD and its management. The VNFD describes all required compute, network, storage resources, scalability and operating workflows, and also supports different types of deployments, e.g. small and big deployments.

HP vOCS (Virtualized Online Charging System) is a typical VNF (Virtualized Network Function). Figure 3 illustrates the VNFD (VNF Descriptor) with some key elements we are using to describe HP vOCS. HP vOCS includes 3 VNF Components, LB (Load Balancer), OCS (Online Charging Server) and OMC (Operation, Maintenance and Configuration). Each VNF Components can be instantiated to multiple VM instances based on the defined instance count in VNFD. For the VNF Component which can be scaled out/in on demand, its scalability is defined in VNFD. Each VNF Component has multiple network interfaces and connect to virtualized networks, virtualized networks are defined in VNFD too, and they will be created while the vOCS is created (instantiate). Figure 6 illustrates the HP Helion Dashboard to show a new vOCS is created from scratch based on a VNF Descriptor with small deployment flavor through an instantiate operation.

While the vOCS is created (instantiated), VNEM will install and configure each VM with predefined artifacts, and then start the application on the VM. Those operations are mapped to lifecycle operations.

The VNEM can manage a full lifecycle of a VNF from VNF on-board, instantiate, configure, scale in/out, start/stop, terminate and VNFD demission. Figure 4 illustrates the VNF lifecycle managed by VNEM.

Figure 6 also illustrates HP Helion Dashboard with vOCS operations (Instantiate, Scale in/out, Terminate), e.g. the scale out operation to create a new VM. The scale out operation can be triggered by VNEM, or by an external NFV Orchestrator, it can be automatic based on the runtime data of vOCS, e.g. the traffic throughputs, or it can be manually triggered.

VNEM provides a CLI (Command Line Interface) to operate on the VNF and its VNF instances.

One VNEM can manage multiple VNF and its multiple VNF instances. 


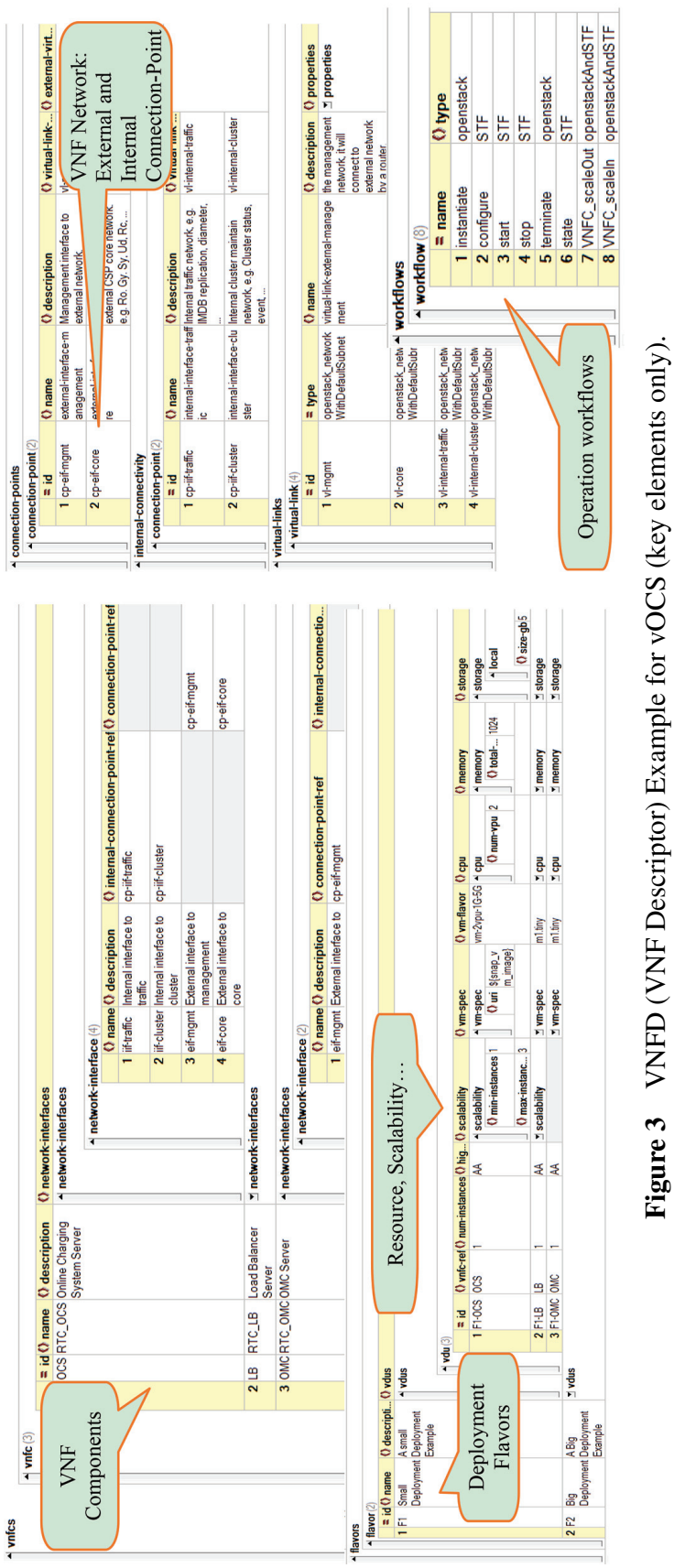






Figure 4 VNF lifecycle with VNEM.

Once a VNF is instantiated, a VNF instance will be created based on the specified VNFD and deployment flavor, and a new VNFR (VNF Record) will be created. The VNFR includes the logical and physical resources allocated for the VNF instance, and contains sufficient information (e.g. IP addresses of VM) to manage and change the deployed VNF instance later on. Figure 5 illustrates an example of VNFR for vOCS.

The STF workflow implementation is organized by VNFC (VNF component). All the VNF related context, including VNFD, VNFR, current VNFC instance, and configuration are available to be accessed as input parameters and can output any result to the context. A STF workflow includes a set of task sequences. It comes with a template that includes STF flows and artifacts. This template is filled and rendered by the different elements that constitute the VNF context, such as VNFC instance, VNFR, VNFD. The typical task types can be remote shell, remote copy to or from... 


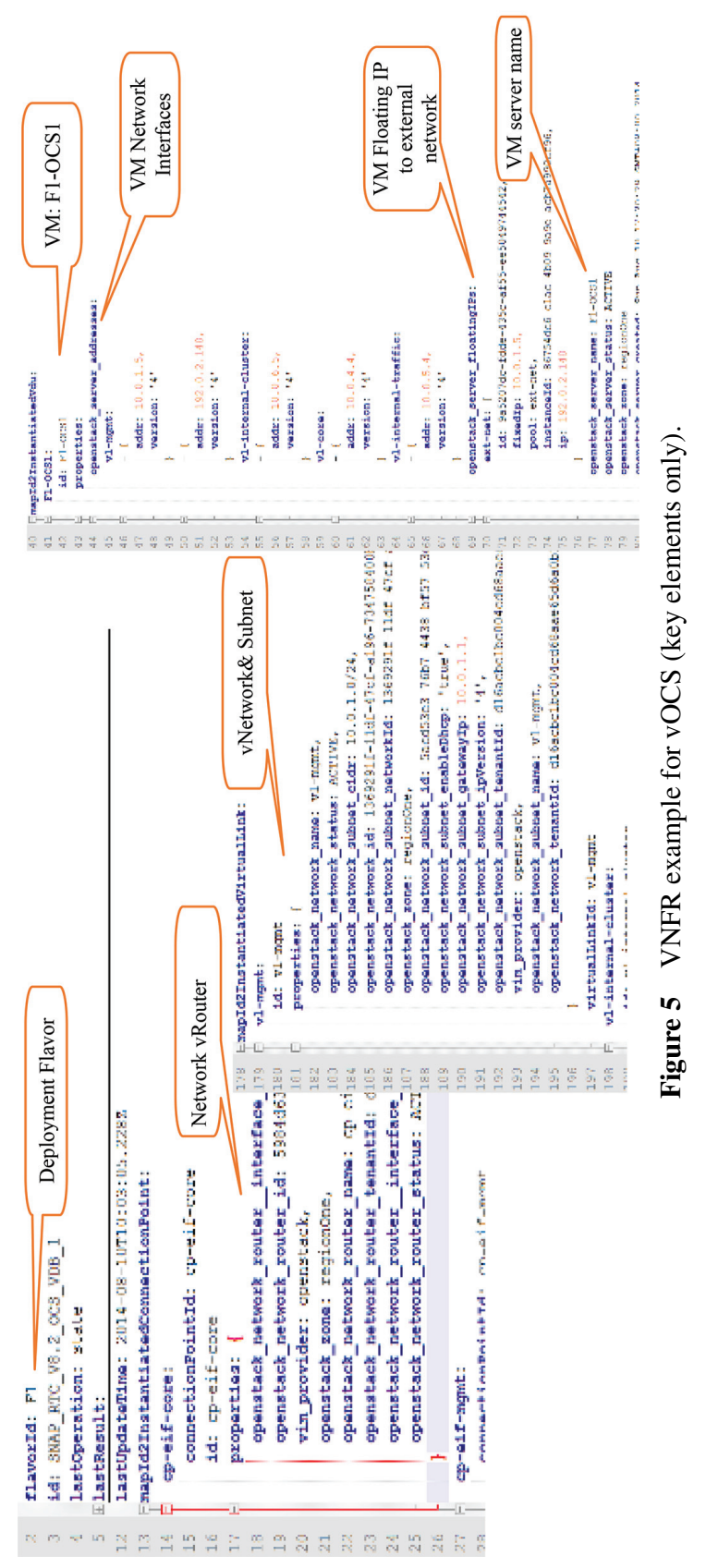




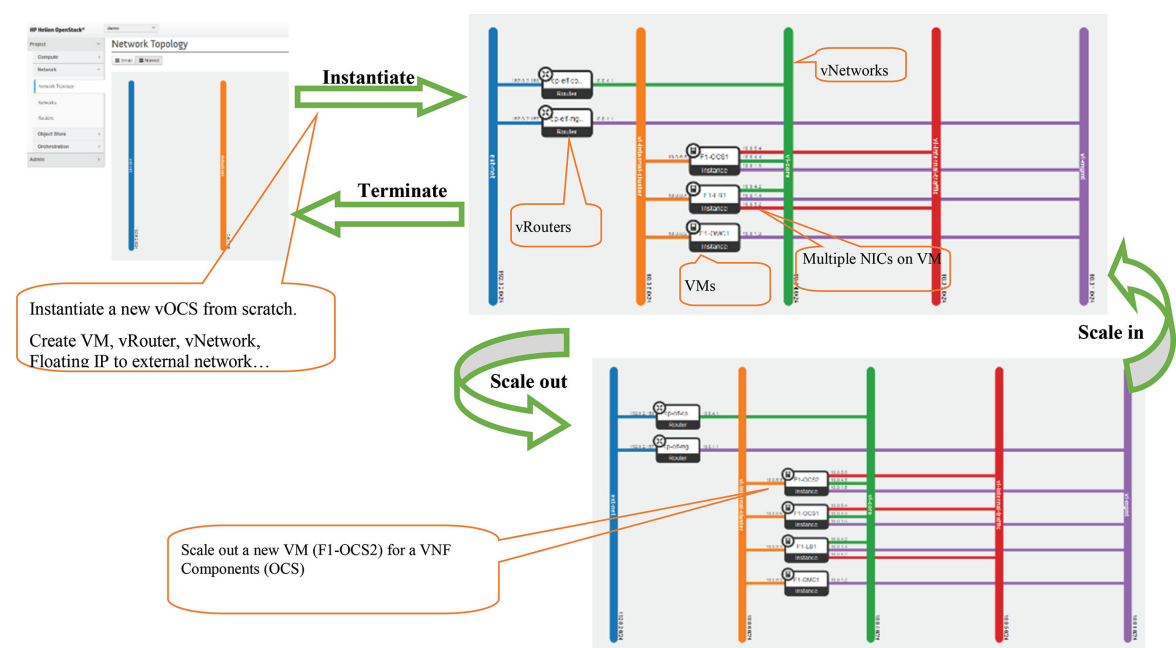

Figure 6 HP Helion dashboard with vOCS operations (Instantiate, Scale in/out, Terminate).

\section{Evidence the Solution Works}

We have done a PoC (Proof of Concept) with HP OCS, including a vOCS VNF, a VNEM prototype and HP Helion Openstack community edition. Figure 7 illustrates the POC architecture.

The POC (Proof of Concept) environment is built on a HP Helion Openstack platform, running on HP BL blade bare metal servers, and the hypervisor is KVM. The VNEM is running on a Linux VM server. VNEM interacts with HP Helion with Openstack nova and neutron API to create the VM and virtualized networks based on the VNF descriptor, automatically. Once the VNF required virtualized resources are created (instantiated), VNEM interacts with the VMs with SSH based workflow and tasks to execute other operations.

\section{Competitive Approaches}

$\mathrm{NFV}$ is an emerging technology where management and orchestration is the hot topic, but today most of the discussions are focused on the NFV Orchestrator part. The evolution of EM and VNF Manager are up to individual VNF teams, no specifications, no standard and no toolkit or product are available for VNF developers to use. It becomes a major challenge to transform existing Network Function to a Virtualized Network Function and support ETSI NFV MANO (Management and Orchestration). Our VNEM solution facilitates this 


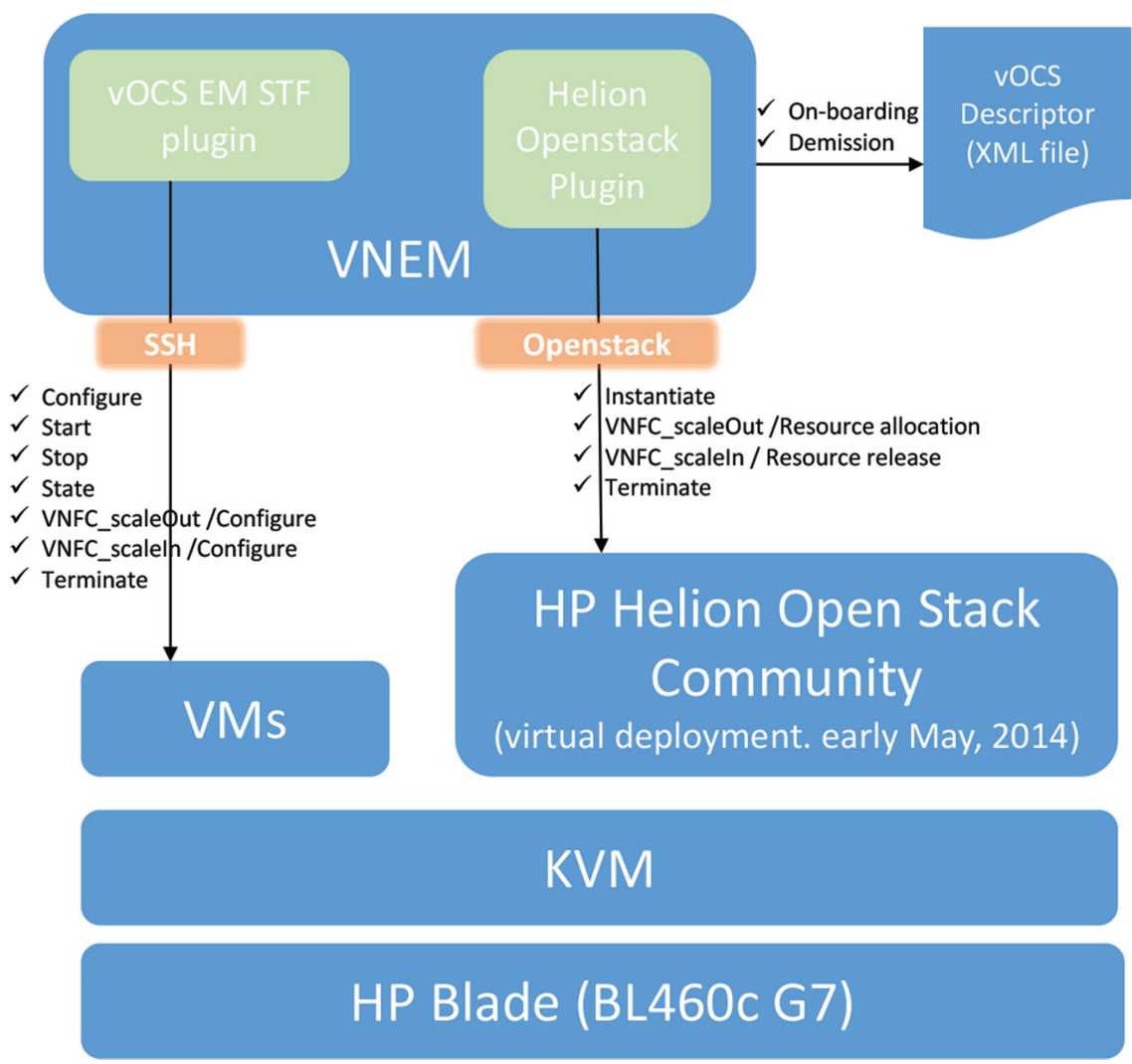

Figure 7 VNEM POC arch.

transformation and makes it easier and quicker. We have demonstrated the VNEM PoC to many experienced people and got very positive feedback. So far, we are not aware of any Network Function independent solution for this transformation.

\section{Current Status}

We have implemented a prototype during our PoC, which is illustrated in Figure 7. The Openstack environment is HP Helion virtual deployment edition released in May 2014.

The POC covered all scenarios illustrated in Figure 4. Most of the NFV MANO (Management and Orchestration) scenarios are implemented and 
demonstrated, for example, automatic deployment with predefined VNFD, create a vOCS within minutes, scale out/in within minutes ... We also produced a demo video which is available at: http://pan.baidu.com/s/1sjDgP1z

The video demonstrates the VNF Descriptor, lifecycle and the operations illustrated in Figure 4 with the VNEM CLI, the HP Helion dashboard and the vOCS Component VM interactions.

From overall NFV MANO architecture perspective, VNEM acts as a VNF Manager, and needs to be integrated with an NFV Orchestrator, such as HP NFV Director.

Thanks for the easy and efficient integration approach provided by VNEM, recently, we transformed an HPPCRF (3GPP Policy and Charging Rule Function) product to become a virtualized PCRF (vPCRF) in less than a month. This vPCRF within an integrated vEPC (3GPP Evolved Packet Core) solution was demonstrated in Mobile World Congress 2015. Both vOCS and vPCRF are integrated with HP NFV Director.

We also presented a paper in ICIN15 (The 18th International Conference on Intelligence in Next Generation Networks), and the presentation triggered major interest. Subsequently, in ETSI NFV, new contributions regarding generic VNF Manager were proposed and approved.

\section{Next Steps}

We have started to share this work and the actual code within our organization so it helps educate other engineering teams on NFV and the work can be reused to accelerate NFV implementations and support in different product lines. This work can be leveraged to productize at Platform level.

Regarding next steps, we are planning to improve the VNEM with more production features, such as:

- VM anti-affinity to support high availability, allowing same VNF Component VM not being created in a same bare metal server to avoid single point HW failure

- A general KPI (Key Performance Index) mechanism described in VNF Descriptor to let VNEM collect real time KPI data to be used for auto scalability and monitoring;

- A scalability decision engine as an embedded component in VNEM.

- A visual VNF instance deployment and monitoring User Interface.

- A VNF Descriptor GUI Editor 
- A generic open interface between VNEM and NFV Orchestrator, which is the Or-Vnfm reference point in Figure 1, to enable VNEM be integrated with 3rd party NFV Orchestrator.

\section{References}

[1] NFV specifications, ETSI http://www.etsi.org/technologies-clusters/ technologies/nfv

[2] HP Helion: http://helion.hpwsportal.com

\section{Biographies}

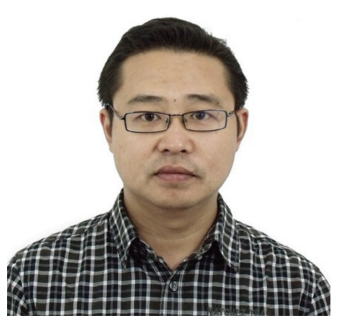

W. Bo is from Communications \& Media Solutions (CMS) Business Unit within Hewlett Packard Enterprise (HPE), who addresses the needs and create new business models, deliver new services and improve customer's satisfaction while achieving operational efficiencies for Communication Service Providers (CSP).

As Master Solution and System Architect for the Hewlett Packard Enterprise Services, Bo has more than 19 years of experience in the Telecommunications, IT, Software and Service industry. Bo has provided technical leadership, recommendations and innovation to leading-edge Pan-HP multi-technology products, solutions and deliveries. Bo performs the role of Chief Solution Architect for 2 Products, SNAP (Subscriber, Network and Application Policy) RTC (Real Time Charging) and UPM (Unified Policy Manager), and a Solution Architect for eIUM (Enhanced Internet Usage Manager) Product, driving innovations, visions and strategies for RTC, UPM and eIUM products, HP CMS, pan HP and HP's customers. 


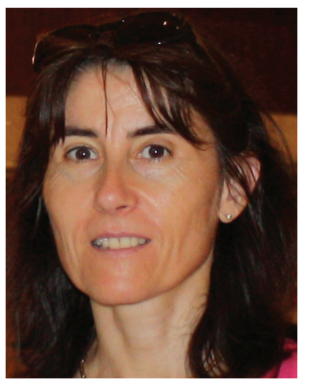

O. Marie-Paule is a seasoned HP executive, bring over 25 years of telecom experience. She has deep expertise in both the networking and IT environments, NFV, SDN and M2M/IoT.

Marie-Paule is Distinguished Technologist for HP CMS, Communication and Media Solution organization, focused on customer innovation and emerging trends in the communication industry. She leads the technology discussions for NFV (Network Function Virtualization), M2M, Analytics, Cloud. She seats on ETSI, ATIS, IEEE and other standard bodies. She is Vice-chairman of ETSI NFV ISG, seats on the Technical Steering Committee, is a Vice Chair of TST working group (testing, interoperability and Opensource), rapporteur of SDN work item, and Vice Chair in IEEE SDN. She participates in European Commission SDN-NFV task force, and is also active on M2M \& IoT, within ETSI and OneM2M, rapporteur of TG28 low throughput network work items, working with new players such as Sigfox or LORA technologies. She holds a few patents and is also a frequent industry speaker and editor in professional magazines and blogs, incl HP Telecom IQ.

Marie-Paule prior responsibilities include managing HP's worldwide VoIP program, HP's wireless LAN program, and HP's Service Delivery program. Since joining HP in 1987, she has held positions in technical consulting, sales development and marketing in Europe and in the Americas. Those roles have focused on strategic and operational responsibility for Networking, IT and operations in the telecom domain.

Marie-Paule holds a master's degree in Electrical Engineering from Utah State University and business education from INSEAD, Paris. Prior to joining HP, Marie-Paule spent five years with France-Telecom/Orange research and development labs, defining architecture and value-added services launch for corporate customers. She enjoys skiing and outdoors in general. 